\title{
Screening of transverse ac fields and influence of supercurrent on NMR Knight shift in high- $T_{c}$ crystals
}

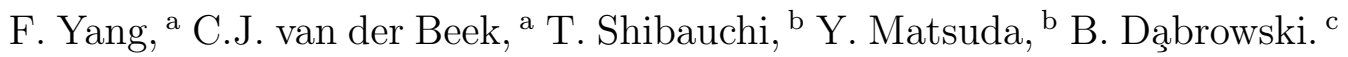 \\ ${ }^{a}$ Laboratoire des Solides Irradiés, CNRS-UMR 7642 \& CEA/DSM/DRECAM, Ecole Polytechnique, 91128 Palaiseau, France \\ b Department of Physics, Kyoto University, Sakyo-ku, Kyoto 606-8502, Japan \\ ${ }^{\mathrm{c}}$ Northern Illinois University, Faraday Hall W216, De Kalb, IL 60115, U.S.A
}

\begin{abstract}
In the Nuclear Magnetic Resonance (NMR) configuration with perpendicular constant (dc) field and radiofrequency (rf) magnetic fields, a strong screening supercurrent is generated by the rf field. In order to estimate this current which may influence Knight shift data, we performed a model experiment in the NMR configuration on a $\mathrm{YBa}_{2} \mathrm{Cu}_{4} \mathrm{O}_{8}$ single crystal. The differential magneto-optical imaging technique is used to determine the ac screening current distribution in the sample. We find that at $T>20 \mathrm{~K}$, NMR experiments can be straightforwardly interpreted. At $T<20 \mathrm{~K}$, the rf field is completely excluded. In this situation, NMR only probes the crystal edges; moreover, the Knight shift may be affected by the supercurrent flow.
\end{abstract}

Key words: Superconductor; Knight-shift; d-wave; Volovik effect.

PACS: $74.25 . \mathrm{Qt}, 74.25 . \mathrm{Sv}, 74.25 . \mathrm{Nf}$

\section{Introduction}

The NMR Knight shift $K_{s}$ probes the density of states (DOS) at the Fermi surface. In a superconductor, measurements of the $K_{s}$ at low temperature can thus probe the symmetry of the superconducting order parameter. However, in the NMR configuration: (i) The supercurrent flow is not uniform and it changes with temperature. (ii) For superconductors with gap nodes, in the presence of a magnetic field, the DOS at the Fermi surface for quasi-particles is Doppler-shifted by the supercurrent. An example is the shift of $N_{s}(E) \sim \sqrt{H}$ due to the vortex currents, known as the Volovik effect [1].

Here, we determine the distribution of the screening supercurrent and its possible effect on the Knight shift by Magneto-Optical (MO) imaging of a $\mathrm{YBa}_{2} \mathrm{Cu}_{4} \mathrm{O}_{8}$ crystal in the orthogonal field configuration proper to NMR.

\section{Experiment}
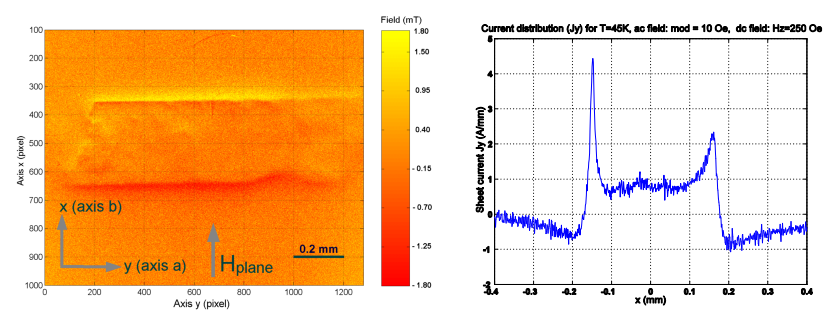

Fig. 1. Left: DMO image of the perpendicular component of the induction, induced by $H_{\text {plane }}$, obtained at $T=45 \mathrm{~K}$ with a 10 Oe square wave modulation along the $b$ axis. Right: Corresponding sheet current $J_{y}(x)\left(\mathrm{Amm}^{-1}\right)$ at $T=45 \mathrm{~K}$.

The sample is a stripshaped $\mathrm{YBa}_{2} \mathrm{Cu}_{4} \mathrm{O}_{8}$ single crystal $\left(T_{c}=82 \mathrm{~K}\right)$ of dimensions $1220 \times 328 \times$ $88 \mu^{3}$. We reproduce the field configuration employed in NMR: a dc field of 250 Oe is applied along the $c$ axis and a periodic transverse field is applied 
along the $b$ axis. The penetration of the periodic field was imaged using the Differential Magneto-Optical (DMO) technique. A transverse field of 10 Oe was applied, then $10 \mathrm{MO}$ images were acquired and averaged. The transverse field was then removed, another 10 images were collected, averaged and subtracted from the first average. This procedure was repeated 10 times. We thus obtained images of the flux penetration into the crystal due to a 10 Oe square wave transverse field $H_{\text {plane }}$ of frequency $f=$ $50 \mathrm{mHz}$. We suppose that the dynamics of flux lines do not change significantly with the frequency.

Figure 1 shows a DMO image of the perpendicular component of the induction, induced by $H_{\text {plane, }}$ obtained at $T=45 \mathrm{~K}$. Measurements were done for temperatures between $15 \mathrm{~K}$ and $80 \mathrm{~K}$.

The screening current distribution $J_{y}(x)$ is calculated by inversion of the Biot-Savart law [2]. Three regimes are clearly manifest: At $T>65 \mathrm{~K}$, current flow is uniform. At $T<20 \mathrm{~K}$, one has the critical state due to bulk flux pinning. The current flows in the regions penetrated by the ac field only. At intermediate temperature $20 \mathrm{~K}<T<65 \mathrm{~K}$, current flows only at the sample edges due to the geometrical barrier effect (Fig. 1).

From the current distribution, we reconstruct the magnitude of the transverse field $H_{x}$ modulation normalized to $H_{\text {plane }}$ (Fig. 2). This corresponds to the local probing field in NMR. For $T>20 \mathrm{~K}$, the transverse field is almost homogenous, the whole sample contributes to the NMR signal. For $T<20$ $\mathrm{K}$, only a thin surface layer contributes to the NMR signal.

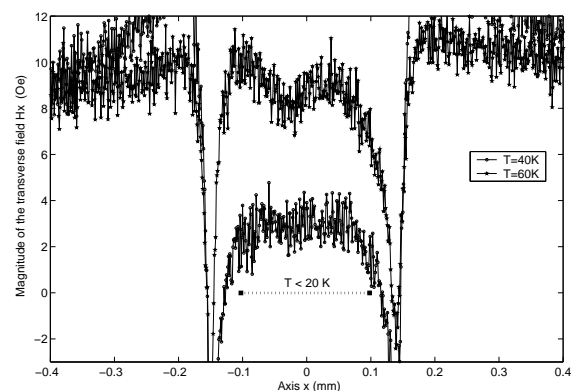

Fig. 2. Magnitude of the transverse field $H_{x}$ modulation normalized to $H_{\text {plane }}$ : circle $40 \mathrm{~K}$, star $60 \mathrm{~K}$.

\section{Discussion of the Volovik effect on NMR Knight shift measurements}

For a d-wave superconductor, in the absence of a magnetic field, $N_{s}(E) / N_{0}=\frac{2}{\pi} \frac{E}{\Delta_{0}} K\left(\frac{E}{\Delta_{0}}\right)$, where
$N_{s}(E)$ is the superconducting density of states; $N_{0}$ is the density of states at the Fermi surface in the normal phase, $\Delta_{0}$ is the maximum gap and $K(x)$ is the complete elliptic integral. For small $\frac{E}{\Delta_{0}}$, or when $T<0.4 T_{c}$, numerical evaluation shows that $N_{s}(E)$ increases linearly with $E[3]$. The application of a magnetic field results in a shift of the quasi-particle energy levels due to the superflow: $E_{k}=E_{k}^{0}+\mathbf{p}_{\mathbf{k}} \cdot \mathbf{v}_{\mathbf{s}}$. The modification of the DOS at the Fermi level due to the screening current is estimated as $\frac{N(0, j)}{N_{0}}=$ $\frac{\left\langle\mathbf{p}_{\mathbf{F}} \cdot \mathbf{v}_{\mathbf{s}}\right\rangle}{\Delta_{0}} \approx \frac{1}{2 \sqrt{2}} \frac{T_{F}}{T_{c}} \frac{v_{s}}{v_{F}}$, where \langle\rangle means the average over the Fermi surface, $\mathbf{p}_{\mathbf{F}}$ is the Fermi momentum, $v_{F}$ is the Fermi velocity, $v_{s}$ is the superflow velocity, $\Delta_{0} \approx 2 k_{B} T_{c}$ (weak-coupling limit).

For example, in our crystal at $\mathrm{T}=45 \mathrm{~K}$, the sheet current $j_{d}$ (integrated over the sample thickness $d=88 \mu \mathrm{m}$ ) at the edges is $4.5 \times 10^{3} \mathrm{Am}^{-1}$. The maximum current density $j=5 \times 10^{7} \mathrm{Am}^{-2}$. At $T<20$ $\mathrm{K}$, the current density reaches $10^{8} \mathrm{Am}^{-2}$, which results in $v_{s} \approx 5 \mathrm{~ms}^{-1}$, to be compared to $v_{F} \approx$ $1.2 \times 10^{5} \mathrm{~ms}^{-1}[4], T_{F} \approx 4000 K$, i.e. a Doppler shift of $0.15 \%$. This is to be compared to the Volovik effect [1]: $N(0, H) / N_{0}=h\left\langle p_{F} \cdot v_{s}\right\rangle / \Delta_{0} \approx K \sqrt{H / H_{c 2}}$, with $K \approx 1, H_{c 2}=100 \mathrm{~T}$, and $H=1 \mathrm{~T}(\mathrm{NMR}$ experimental condition) yields $N(0, H) / N_{0}=10$ $\%$. The effect due to the screening current due to $H_{\text {plane }}$ can thus be ignored for our $\mathrm{YBa}_{2} \mathrm{Cu}_{4} \mathrm{O}_{8}$ crystal. But for other high- $\mathrm{T}_{c}$ superconductors, e.g., $\mathrm{Bi}_{2} \mathrm{Sr}_{2} \mathrm{CaCu}_{2} \mathrm{O}_{8}$, in which screening currents of the order of $10^{10} \mathrm{Am}^{-2}$ are attained, the Doppler shift due to screening current may strongly influence the $K_{s}$ below $15 \mathrm{~K}$.

For a $d$-wave superconductor like $\mathrm{YBa}_{2} \mathrm{Cu}_{4} \mathrm{O}_{8}$, a linear dependence of the Knight shift on temperature should be observed for $0.1 T_{c}<T<0.4 T_{c}$. While the Volovik contribution may lead to a nonvanishing Knight shift for $T \rightarrow 0$, the Doppler shift due to the screening current may lead to a nonlinear dependence of the $K_{s}$ on temperature.

\section{References}

[1] G.E. Volovik, JETP Lett. 58 (1993) 469.

[2] T.H. Johansen et al, Phys. Rev. B 54, 16264 (1996)

[3] H. Won and K. Maki, Phys. Rev. B 49, 1397 (1994)

[4] M. Chiao et al, Phys. Rev. Lett. 82, 2943 (1999) 